\title{
COMPUTATIONAL ANALYSIS OF PASSIVE MIXING IN T-MICROMIXER WITH NON-NEWTONIAN BLOOD
}

\section{SULEKH TOKAS \& MOHAMMAD ZUNAID}

Department of Mechanical Engineering, Delhi Technological University (DTU), Bawana Road, Delhi, India-110042

\begin{abstract}
Micromixers are an essential integral part of the Lab-on-chip (LOC) devices which carry importance in the domain of chemical, biomedical and biochemical applications. The flow and mixing behavior of fluid changes drastically when the length size is scaled down to the order of microns due to the dominance of viscous forces as compared to the macroscale where the inertial forces influence the flow. The present study examines the flow characteristics and passive mixing behavior in a simple T-micromixer with Newtonian fluid water and Non-Newtonian blood having an aspect ratio (width/height of the channel) 2 using computational fluid dynamics (CFD) for a wide range of Reynolds number (2 $\leq$ Re $\leq 320)$ for water and the corresponding mass flow rates of blood. The Carreau-Yasuda model is employed to model the Non-Newtonian rheology of blood used in the simulation, whereas water is considered to be Newtonian fluid possessing constant viscosity. The flow and mixing behavioris different for water and blood. It is seen that initially, mixing efficiency for water decreases with an increase in the Reynolds however, an increase in mixing performance is observed with a further increase of Reynolds number. In contrary to the Newtonian fluid water, a continuous decreasing trend in the mixing efficiency is visible with an increase in the mass flow rate for blood analysis. The maximum mixing efficiency of the micromixer with water is $31.9 \%$, whereas for blood it is $34.5 \%$. Finally, the pressure drop in the mixer between inlets and outlet are studied for both blood and water as it is an important parameter linked to the requirements of pumping power.
\end{abstract}

KEYWORDS: T-Micromixer, Newtonian Fluid, Non-Newtonian Fluid, Blood, Mixing Efficiency, Pressure Drop

Received: Jun 08, 2020; Accepted: Jun 28, 2020; Published: Aug 29, 2020; Paper Id.: IJMPERDJUN2020944

\section{INTRODUCTION}

The study of fluid flow at the microscale, i.e. the length scale of the systems being of the order of micrometers is termed as microfluidics. The concept of miniaturization of various chemical, biochemical, and biological analysis carries enormous advantages. First, there is a significant amount of reduction in the requirement of the sample size. For example, downscaling the linear scale by a factor of $10^{4}$ will reduce the volume requirements by the order of $10^{12}$ and such a lower volume of fluid helps in fast analysis and diagnosis. Second, shrinking down the actual size of chemical/ biological laboratories to the size of the chip will not only bring down the cost associated with set-up and running the laboratory by remarkable amounts but it also makes the laboratory compact and portable.The basic micromixer is the $\mathrm{T}$-junction type mixer and extensive work has been reported describing the three types of flow regimes in this type of micromixer. Several early work in the field of micromixers involved the fabrication of microchannels using silicon. Koch et al.[1] compared two basic micromixers and characterized the mixing using the ammonia in water solution and phenolphthalein solution. Veenstra et al.[2] proposed a novel silicon-based micromixer which produced effective mixing of water and phenolic solution. Gobby et al.[3] studied the mixing pattern in a simple T-mixer for non-reactive gases possessing different viscosities using CFD simulations. The 
effect of various operating and design parameters like aspect ratio (height/width ratio of the main mixing channel), inlet flow rates, angle of the inlet channels, etc was studied. It was found that at low flow rates, the mixing length required is less. Also, more accurate results were obtained in 3-dimensional simulation as compared to the results in a 2-dimensional study. Bothe et al.[4] studied the heat transfer and mixing performance in T-microreactor. It was concluded that reactions at the micro-scale provide better thermal control and chemical reactions take place at a faster rate. They also used CFD simulations to understand the three types of flow regimes seen in the T-micromixer and found that engulfment zone flow provided higher mixing. Wong et al.[5] investigated the mixing performance of T-micromixer experimentally and numerical simulations were used to get an insight into the flow characteristic within the microchannel. Micro T-mixer was fabricated using the silicon substrate which was attached on a Pyrex glass plate. It was found that a pressure of 5.5 bar at inlets was sufficient to mix the two fluids completely within a fraction of a second for a microchannel having a hydraulic diameter of $67 \mu \mathrm{m}$. Soleymani et al.[6] utilized CFD to study the flow and mixing characteristics and proposed optimized results for effective mixing in the liquid phase. It was concluded that secondary flow and vortices are important for obtaining excellent qualitative mixing. Depending on the value of Reynolds number for flow through the main microchannel, the flow was characterized in three different regimes, namely stratified flow, vortex flow, and engulfment flow. A significant rise in the mixing performance was witnessed at higher Reynolds number ( $\operatorname{Re} \geq 130)$ due to the flow entering the engulfment regime where asymmetrical path lines were observed and finally, an optimized TT-mixer was presented for effective mixing in the liquid phase.

Andreussi et al.[7] performed an in-depth examination of the flow regimes in a simple-T micromixer using direct numerical simulations (DNS) which are considered to be a method of great accuracy. The flow patterns were investigated on channels with three different aspect ratios (width to height ratio of the main mixing channel), i.e. $0.75,1 \& 2$. The mixer with aspect ratio 2 showed different evolvement of flow as compared to the other two. As the Reynolds number increased, the flow shifted from vortical to asymmetric and then finally to chaotic at very high Reynolds number. Matsunaga et al.[8] proposed a novel low-cost simulation technique based on particle tracking to obtain mixing behavior in T-micromixer with a rectangular cross-sectional area having high Schmidt number=3600. The results obtained from this model were compared with the results obtained from the backward random walk Monte Carlo simulations. The results were in good agreement. Moreover, it was found that ordinary grid-based simulations did not predict accurate mixing performance at high Reynolds numbers, especially in the engulfment flow regime of the micromixer. Kuo et al.[9] experimentally and numerically investigated performance plasma mixing in three types of micromixers that is square-wave, zig-zag, and curved micromixers completely driven by the capillary action. The square-wave micromixer performed better out of the three due to the presence of stronger secondary flow giving $76 \%$ mixing efficiency in 4 seconds. Moreover, the square-wave mixer was picked for optimization and four different square-wave mixers were examined to predict the best dimensions of the square-wave.

As observed from the presented brief literature review, a thorough study is present which employs various experimental and computational methods to examine mixing behavior for Newtonian fluids, however, less work is available considering blood as Non-Newtonian in a T-micromixer. Since many applications involve blood to be used in the micro total analysis system( $\mu$-TAS), the present work presents a thorough analysis of mixing and flow patterns to understand the shear-thinning behavior of blood in microchannels. 


\section{METHODOLOGY}

\section{Computational Modeling \& Governing Equations}

The basic T-micromixer consists of three major parts that are, inlet channels, the T-junction, and the main mixing channel as depicted in figure 1 . The inlet channels of the micromixer have $100 \mu \mathrm{m} \times 100 \mu \mathrm{m}\left(\mathrm{W}_{\mathrm{i}} \times \mathrm{H}\right)$ square cross-section whereas the outlet has a rectangular cross-section of $200 \mu \mathrm{m} \times 100 \mu \mathrm{m}\left(\mathrm{W}_{0} \times \mathrm{H}\right)$. The aspect ratio which is defined as the width to height ratio of the microchannel is 2 for the main mixing channel and 1 for the inlet channels. The axial length of the Tmixer is fixed at $\mathrm{L}_{t}=3000 \mu \mathrm{m}$ or $3 \mathrm{~mm}$. Also, the Total length of the inlet channels $\left(\mathrm{L}_{\mathrm{i}}\right)$ is considered to be $900 \mu \mathrm{m}$.

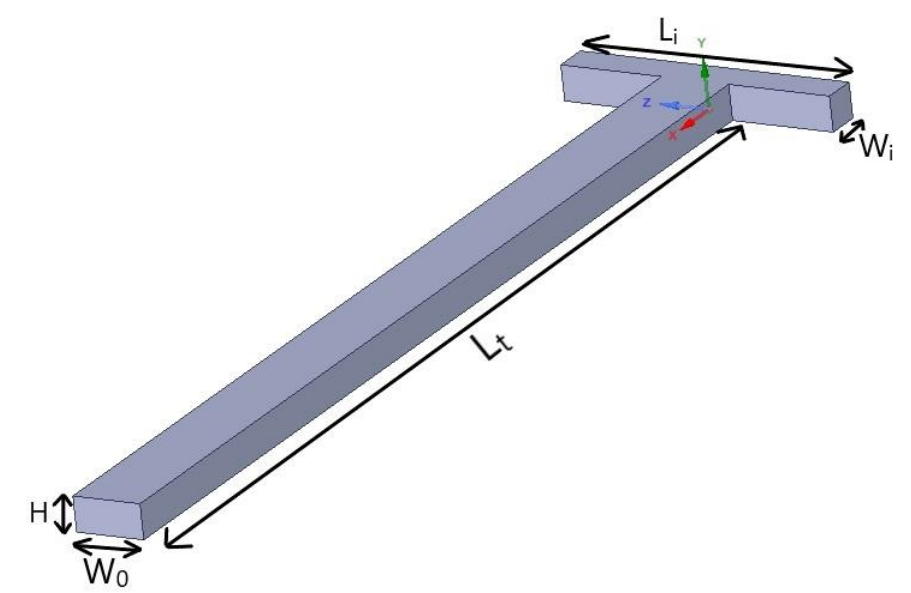

Figure 1: 3-Dimensional Flow Domain of T-Micromixer.

The non-linear governing partial differential equations of momentum, continuity, and species transport are discretized using the finite volume method and solved numerically with the CFD solver of ANSYS fluent. Both the fluids under consideration are considered to be incompressible and the flow is assumed to be steady. The governing equations are:

\section{Continuity}

$$
\frac{\partial u}{\partial x}+\frac{\partial v}{\partial y}+\frac{\partial w}{\partial z}=0
$$

\section{Navier-Stokes Equation}

$$
\rho(\vec{u} . \nabla) \vec{u}=-\nabla p+\nabla \cdot \vec{t}
$$

Where ${ }^{\nabla}$ represents the Del operator,

$$
\nabla=\frac{\partial}{\partial x} \hat{i}+\frac{\partial}{\partial y} \hat{j}+\frac{\partial}{\partial z} \hat{k}
$$

And ${ }^{\rho}$ is the density of the fluid, $\mathrm{p}$ is the static pressure, ${ }^{\vec{\tau}}$ is the stress tensor given by:

$$
\vec{\tau}=\eta\left(\nabla \vec{u}+\nabla \vec{u}^{t}\right)
$$

Here tis the transpose operation and ${ }^{\eta}$ is the apparent viscosity of the fluid. The viscosity is a physical property of the fluid which is constant in the case of Newtonian fluids whereas, for Non-Newtonian fluids, ${ }^{\eta}$ is shear rate dependent 
and thus is governed by various models depending on the nature of the fluid. In the current analysis, the Carreau-yasuda model is used to capture the blood flow dynamics which have been utilized by several researchers in the past[10]-[12]. According to the Carreau-Yasuda model, shear rate dependent viscosity is given by:

$$
\eta(\hat{\gamma})=\eta_{0}+\left(\eta_{0}-\eta_{\infty}\right)\left[1+\lambda(\hat{\gamma})^{a}\right]^{(n-1) d_{a}}
$$

Where $\eta_{m}, \eta_{0}, \lambda, n$ and a are infinite shear rate viscosity, zero shear viscosity, characteristic relaxation time, flow index, and an empirical constant, respectively. Here, $\eta_{o}=0.1600 \mathrm{~Pa}-\mathrm{s}, \eta_{\infty}=0.0035 \mathrm{~Pa}-\mathrm{s}, \lambda=8.2 \mathrm{~s}, \mathrm{a}=0.64$ and $\mathrm{n}=0.2128$. All the values are referred from the previous work of Abraham et. al[13]. Further, ${ }^{\tilde{\gamma}}$ denotes the scalar shear rate given by:

$$
\dot{\gamma}=\sqrt{2 \times(D: D)}
$$

Where $\mathrm{D}$ is the rate of deformation tensor.

\section{Species Transport Equation}

$$
(\vec{V} \cdot \nabla) C_{A}=D_{A B}\left[\frac{\partial^{2} C_{A}}{\partial x^{2}}+\frac{\partial^{2} C_{A}}{\partial y^{2}}+\frac{\partial^{2} C_{A}}{\partial z^{2}}\right]
$$

Where, $\vec{V}$ is the velocity vector, $C_{A}$ is the concentration of species $\mathrm{A}$ and $D_{A B}$ is the coefficient of molecular diffusivity. The density, dynamic viscosity, and diffusivity are fixed at $998.2 \mathrm{Kg} / \mathrm{m}^{3}, 0.001 \mathrm{~Pa}-\mathrm{s}$, and $2 \times 10^{-9} \mathrm{~m}^{2} / \mathrm{s}$, respectively for water (Newtonian fluid), however for blood (Non-Newtonian fluid), density is $1058 \mathrm{Kg} / \mathrm{m}^{3}$ and diffusivity is $2.8 \times 10^{-10} \mathrm{~m}^{2} / \mathrm{s}$. The velocity inlet boundary conditions are specified at both the inlets with one inlet having species concentration as 1 whereas the other inlet is given the concentration of zero (0). The outlet is given the boundary condition of zero specific pressure. All the walls are assumed to non-porous and no-slip boundary condition is specified for it. The steady-state laminar flow model and species transport model in ANSYS fluent are turned on to understand the flow and mixing behavior. Second-order upwind scheme is used to discretize the continuity, momentum \& species transport equations and the SIMPLEC algorithm is employed for the pressure-velocity coupling. The residual values for all the equations are set to $10^{-8}$ as the convergence criterion.

\section{Quantification of Mixing}

The calculation of mixing performance is done by calculating the mixing efficiency given by the formula:

$$
\begin{aligned}
& M=\left(1-\sqrt{\frac{\sigma^{2}}{\sigma_{\max }^{2}}}\right) \times 100 \\
& \sigma^{2}=\frac{1}{n} \sum_{i=1}^{n}\left(c_{i}-c_{\text {avg }}\right)^{2}
\end{aligned}
$$

Where $\sigma^{2}$ and $\sigma_{\max }^{2}$ are the actual and maximum variance of the mass fraction of the fluid respectively, considered to be at a particular cross-section. A value of $100 \%$ for $\mathrm{M}$ indicates the complete mixing of the two streams whereas $0 \%$ tells that no mixing has taken place. ${ }^{c_{\mathfrak{i}}}$ and ${ }^{c_{\text {avg }}}$ are the value of actual species concentration and average species concentration respectively. $\mathrm{n}$ represents the total number of points considered at the cross-section for the calculation of mixing efficiency. For the current study value of $\mathrm{n}$ is considered to be 700 . 


\section{Mesh Generation and Grid Sensitivity Analysis}

The flow domain of the micromixer is divided into fine hexahedral elements giving a high quality of mesh concerning the orthogonal quality and skewness of the elements as shown in figure 2 (a). The size of the mesh grid elements has huge impact on the results obtained from the numerical solutions. Thus, it becomes important to perform a grid-independent test to minimize the error associated with the size of the element used to generate the mesh. Figure 2 (b) depicts the variation of the mixing index at the outlet of the mixer with the number of hexahedral elements of the mesh for $\mathrm{Re}=266$.

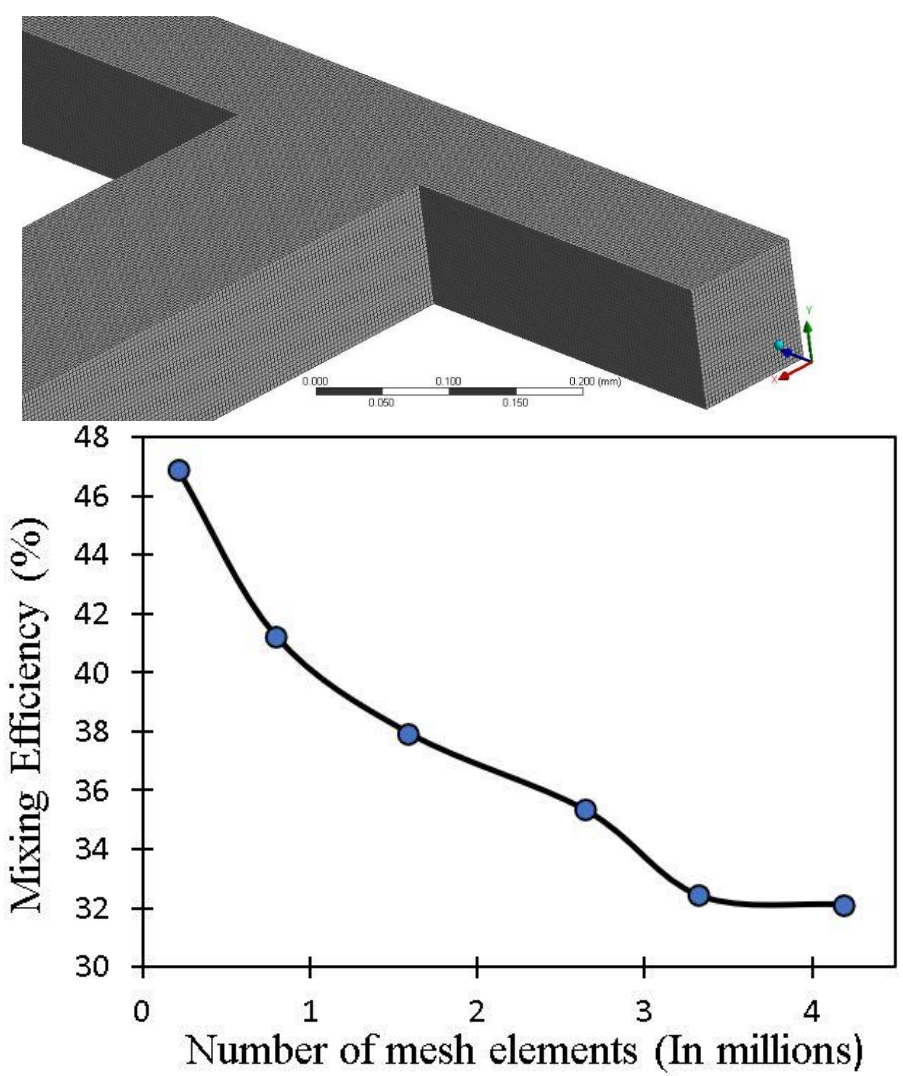

Figure 2: (a). Computational Mesh of Fluid Domain (b) Grid Sensitivity Analysis.

\section{Validation of the Computational Approach}

To validate the computational method employed in the study, the results of mixing efficiency is compared with the existing literature [14] with Newtonian fluid in a simple T-mixer. The results obtained from the current study appear to be in good agreement with that of the results of dundi et al.[14]. Table 1 depicts a comparison of the results for $\mathrm{Re}=266$ at different sections along the length of the mixer. Therefore, the accuracy of the prediction of mixing efficiency is high and the computational approach can be further extended to study mixing in Non-Newtonian mixing.

Table 1: Comparison of CFD Results with the Existing Literature

\begin{tabular}{|c|c|c|}
\hline $\begin{array}{c}\text { Length of Mixing } \\
\text { Channel (X-Direction) }\end{array}$ & $\begin{array}{c}\text { Dundi et al. [14] Mixing } \\
\text { efficiency \% }\end{array}$ & $\begin{array}{c}\text { Current CFD results Mixing } \\
\text { efficiency \% }\end{array}$ \\
\hline $0 \mu \mathrm{m}$ & 5.551 & 6.32 \\
\hline $250 \mu \mathrm{m}$ & 21.28 & 22.5 \\
\hline $500 \mu \mathrm{m}$ & 23.35 & 24.85 \\
\hline $1000 \mu \mathrm{m}$ & 25.39 & 26.2 \\
\hline $1500 \mu \mathrm{m}$ & 26.702 & 28.9 \\
\hline $2000 \mu \mathrm{m}$ & 28.12 & 29.1 \\
\hline $3000 \mu \mathrm{m}$ & 30.72 & 31.1 \\
\hline
\end{tabular}




\section{RESULTS \& DISCUSSIONS}

The mixing index or mixing efficiency calculated at a particular cross-section is a parameter to quantify the mixing phenomenon occurring between the flowing fluids in the microchannel. For Newtonian fluids like water considered in the study, it is observed that initially at low values of Reynolds number the residence time of the flowing fluids is very high which means that they stay in contact inside the channel for a longer period. This results in insufficient time for the molecular diffusion mass transport to occur at the molecular level at the interface of the two fluids and therefore, the mixing index is high at lower values of Reynolds number. However, with an increase in the Reynolds number of flows, the residence time of fluids decreasing which results in a shorter duration of interfacial contact between the mixing fluids. Since the fluids come out of the mixer quickly, the mixing index obtained at the outlet of the channel decreases as seen in figure 3. A decreasing trend is observed in the mixing index with minimum reaching to $3.9 \%$ at $\operatorname{Re}=66$ until the critical Reynolds number, post which the flow enters into the engulfment zone where swirling of fluids or vortices start forming due to the high-velocity collision of entering fluids at the T-junction itself. This results in stretching of the interfacial area as seen in figure 4 which depicts the concentration of species art outlet for $\mathrm{Re}=266$ that enhances the species diffusion mass transport and thus themixing efficiency. The maximum efficiency of $31.9 \%$ is obtained at $\mathrm{Re}=320$ which the highest value of Reynolds number considered in the study.

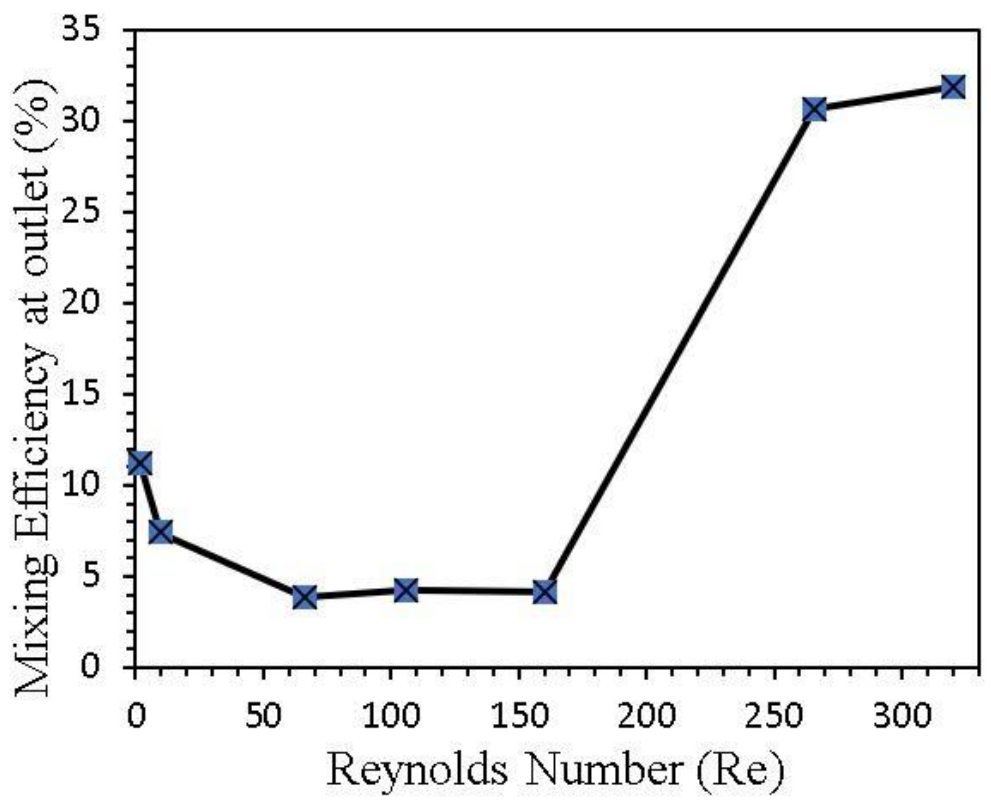

Figure 3: Variation of Mixing Efficiency at the Outlet with Reynolds Number for Water.

The mixing efficiency is computed for a wide range of mass flow rates $\left(5 \times 10^{-5} \mathrm{~kg} / \mathrm{hr} \sim 1.8 \times 10^{-1} \mathrm{~kg} / \mathrm{hr}\right)$ and a completely different for the case of Non-Newtonian blood passive mixing. At low values of mass flow rate, the fluidsremain in contact for a longer period and thus high efficiency of $34.5 \%$ is obtained at $\mathrm{m}=5 \times 10^{-5} \mathrm{~kg} / \mathrm{hr}$ as seen in figure 5. However, with a further increase in the mass flow rate of blood, the mixing index at the outlet of the micromixer decreases sharply due to the insufficient time availability and the fluid coming out at a faster rate. The minimum efficiency obtained for blood is $1.28 \%$ at the highest mass flow rate considered, $\mathrm{m}=1.8 \times 10^{-1} \mathrm{~kg} / \mathrm{hr}$ corresponding to the Reynolds number of 320 in the case of water. 


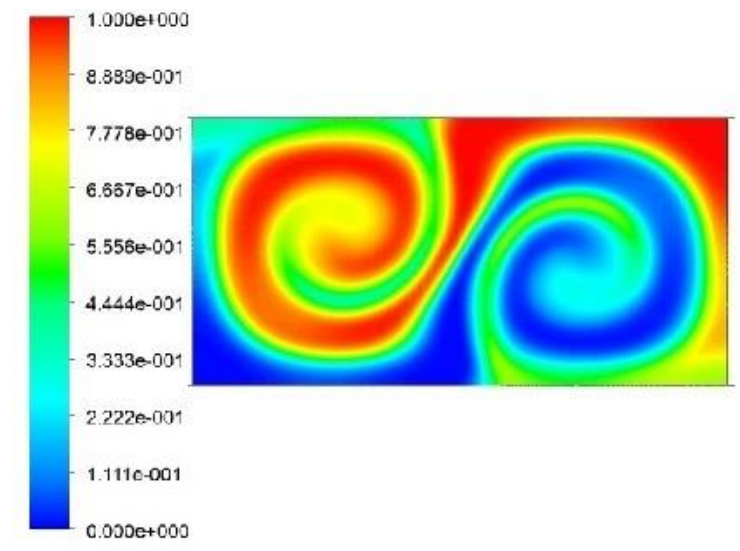

Figure 4: Species Concentration at Outlet of the Micromixer for $\operatorname{Re}=266$ for water.

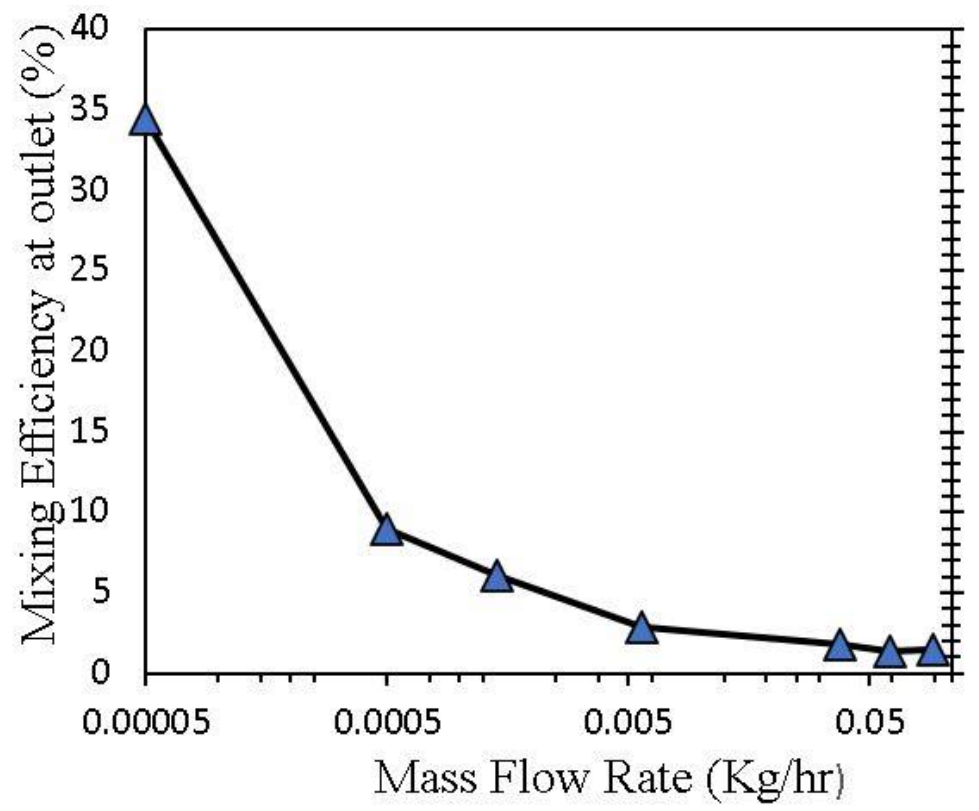

Figure 5: Variation of Mixing Efficiency at the Outlet with Mass Flow Rate for Blood.

To understand the passive mixing in the microchannel, it is crucial to look at the flow pattern for both blood and water separately. Figure 6 (a) \& (b) illustrates the velocity vector on a plane located at the T-junction of the micromixer for water and blood at $\mathrm{Re}=266$ and the corresponding mass flow rate $\mathrm{m}=1.52 \times 10^{-1} \mathrm{Kg} / \mathrm{hr}$ respectively. It can be inferred from figure 6 (a) that the formation of vortices begins at the junction due to the high-velocity collision in the case of Newtonian fluid water which enhances the mass diffusion between the interface. However, the strength of secondary flow or vortex is less in the case of blood. The high viscosity of blood dampens the vortices leading in weak strength of secondary flow which results in poor mixing efficiency even at high flow rates as seen in figure 7 (a) and (b). Figure 7(a) \& (b) illustrates streamlines based on the species concentration at the mid-plane of the inlet channels. 


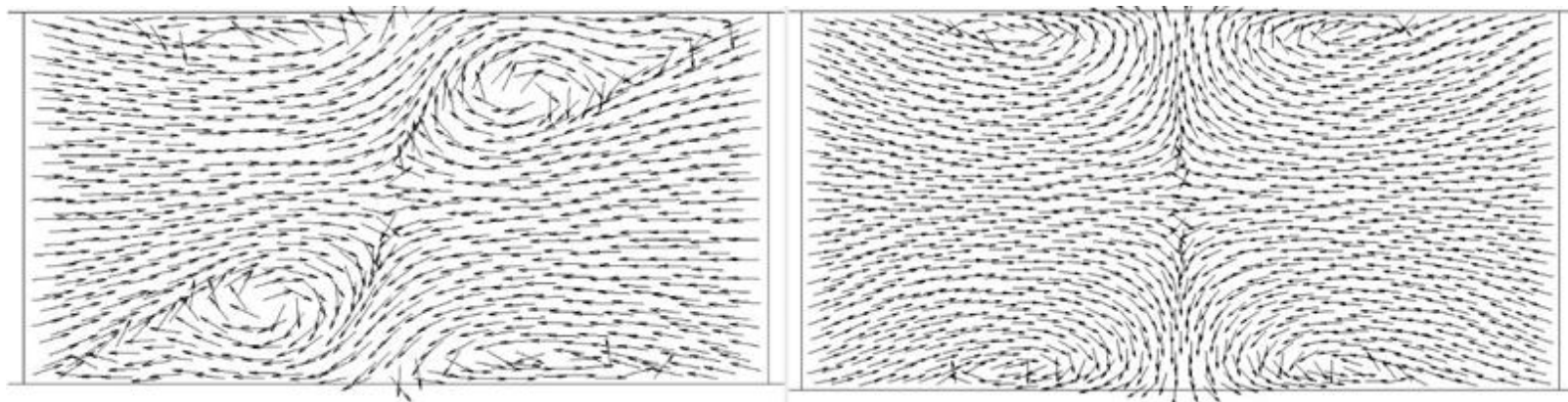

Figure 1: Velocity Vector Plot for (a) Water at $\operatorname{Re}=266$ \& (b) $B l o o d ~ a t ~ m=1.52 \times 10^{-1} \mathrm{Kg} / \mathrm{hr}$.
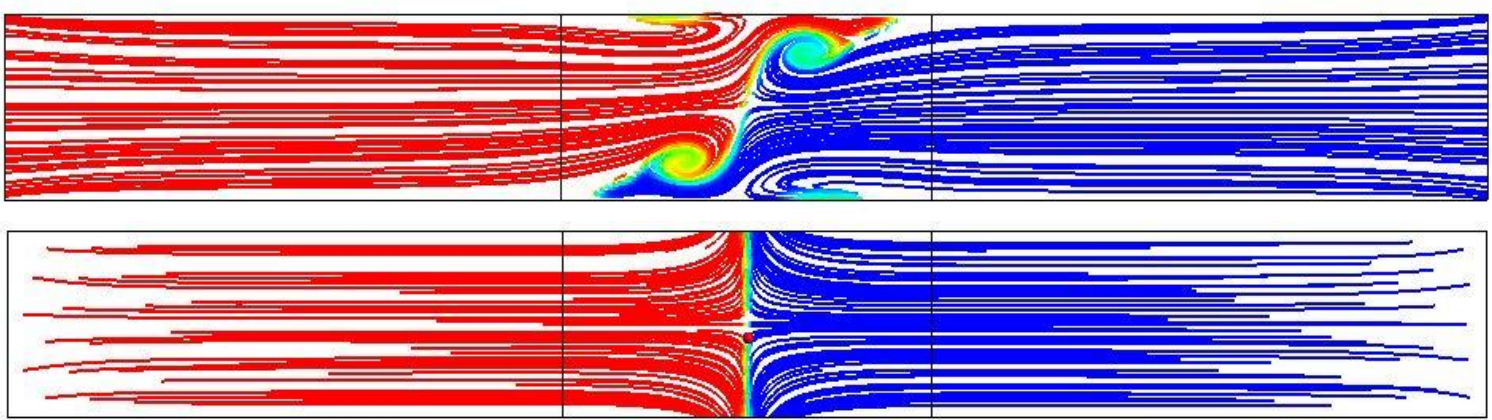

Figure 2: Streamlines Based on the Species Concentration for (a) Water at $\operatorname{Re}=266$ (b) $\operatorname{Blood}$ at $\mathrm{m}=1.52 \times$ $10^{-1} \mathrm{Kg} / \mathrm{hr}$.

This explains the deviation in the mixing index pattern observed between water and blood. The analysis of mixing always requires the study of pressure drop between the inlets and outlet of the micromixer and this becomes more significant in the case of passive micromixers because the pressure drop presents an idea of the power which will be required to pump the fluids from inlets to the outlet. Thus, it becomes essential to investigate the pressure drop in the micromixers which is directly linked with the requirements of input energy. Figure 8 shows the variation of pressure between inlet and outlets with mass flow rate for blood. At low mass flow rate $\left(\mathrm{m}=5 \times 10^{-5} \mathrm{~kg} / \mathrm{hr}\right)$, the pressure drop is 22 pascal which is very less when the pressure drop is compared with $28.49 \mathrm{KPa}$ at the highest mass flow rate of blood. Although the pressure drop is increasing with an increase in the mass flow rate, however, a continuous drop is seen in the mixing efficiency making T-micromixer a bad choice when dealing with high mass flow rate blood applications. Therefore, some modifications in the design of this mixer are needed to enhance the mixing performance and this will be the area of our future work.

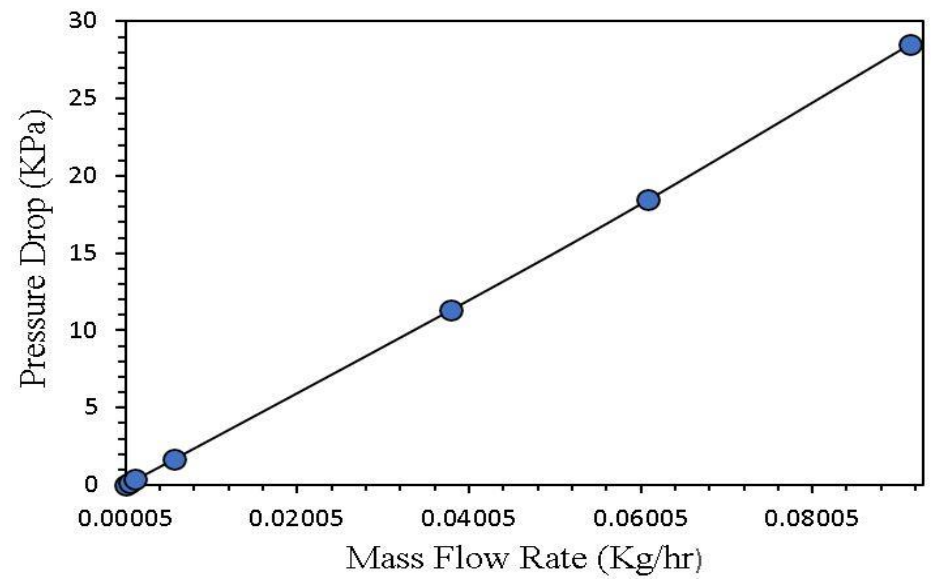

Figure 8 


\section{CONCLUSION}

Flow at microscales is laminar in nature and therefore is dominated by viscous forces which propose a challenge of poor mixing due to the absence of turbulence which enhances the mixing phenomenon. The current study presents the flow and mixing analysis in a conventional T-micromixer for water and blood exhibiting Newtonian and Non-Newtonian characteristics respectively. It was found that at a low value of mass flow rate and the corresponding Reynolds number, better mixing is achieved for the blood as compared to that of water. With an increase in the Reynolds number, the mixing efficiency for the case of Newtonian fluid rises significantly with maximum mixing index of $31.9 \%$ at $\mathrm{Re}=320$ due to the formation of vortices initiating from the T-junction, whereas a decreasing pattern of mixing index is seen with the increase in the mass flow rate for blood with minimum value reaching to as low as $1.28 \%$ making the T-mixer a bad choice to mix blood operating at high values of mass flow rates. The pressure drop between the inlets and outlet of the mixer increases in a linear fashion with an increase in the mass flow rate of blood reaching a maximum value of $28.49 \mathrm{KPa}$ at the highest mass flow rate considered in the analysis. Future work will entail improvements in the T-mixer design for efficient mixing for the case blood at high mass flow rates which is a Non-Newtonian fluid.

\section{REFERENCES}

1. H. Search, C. Journals, A. Contact, M. Iopscience, and I. P. Address, "Two simple micromixers based on silicon," vol. 123, pp. 5-9.

2. H. Search, C. Journals, A. Contact, M. Iopscience, and I. P. Address, "Characterization method for a new diffusion mixer applicable in micro flow injection analysis systems, " vol. 199, pp. 2-6, 1999.

3. Rao, K V Narasimha, Meduri Sitaram, and Naveen Janjanam. "The Effect of Inlet Flow Profile, Carotid Bulb Diameter and Non Newtonian Blood Viscosity on the Wall Shear Stress in a Carotid Artery Bifurcation Model for Transient Flow." International Journal of Mechanical and Production Engineering Research and Development (IJMPERD) 9.5, Oct 2019, 571580

4. D. Gobby, P. Angeli, and A. Gavriilidis, "Mixing characteristics of T-type microfluidic mixers," vol. 126, 2001.

5. D. Bothe, C. Stemich, and H. J. Warnecke, "Fluid mixing in a T-shaped micro-mixer," Chem. Eng. Sci., vol. 61, no. 9, pp. 2950-2958, 2006, doi: 10.1016/j.ces.2005.10.060.

6. S. H. Wong, M. C. L. Ward, and C. W. Wharton, "Micro T-mixer as a rapid mixing micromixer," Sensors Actuators, B Chem., vol. 100, no. 3, pp. 359-379, 2004, doi: 10.1016/j.snb.2004.02.008.

7. A. Soleymani, E. Kolehmainen, and I. Turunen, "Numerical and experimental investigations of liquid mixing in T-type micromixers," Chem. Eng. J., vol. 135, no. SUPPL. 1, 2008, doi: 10.1016/j.cej.2007.07.048.

8. Begam, M. Jannath, and M. Deivanayaki. "An Unsteady Pulsatile Flow of Some MHD Non-Newtonian Nanofluids with Hall Current and Ion Slip Through a Porous Medium." International Journal of Mechanical and Production Engineering Research and Development (IJMPERD) 9.4, Aug 2019, 111-118

9. T. Andreussi, C. Galletti, R. Mauri, S. Camarri, and M. V. Salvetti, "Flow regimes in T-shaped micro-mixers," Comput. Chem. Eng., vol. 76, pp. 150-159, 2015, doi: 10.1016/j.compchemeng.2015.02.017.

10. T. Matsunaga, H. J. Lee, and K. Nishino, "An approach for accurate simulation of liquid mixing in a T-shaped micromixer," Lab Chip, vol. 13, no. 8, pp. 1515-1521, 2013, doi: 10.1039/c3lc41009a. 
11. J. N. Kuо, H. S. Liao, and X. M. Li, "Design optimization of capillary-driven micromixer with square-wave microchannel for blood plasma mixing," Microsyst. Technol., vol. 23, no. 3, pp. 721-730, 2017, doi: 10.1007/s00542-015-2722-1.

12. Sadek, Hasanain Aa, Mohammed J. Khami, and Taleb AS Obaid."Computer Simulation of Blood Flow in Large Arteries by a Finite Element Method." International Journal of Computer Science and Engineering (IJCSE) 2.4 (2013): 171-184.

13. A. Afzal and K. Y. Kim, Flow and mixing analysis of non-Newtonian fluids in straight and serpentine microchannels, vol. 116. Elsevier, 2014.

14. A. Husain, N. Z. Al-Rawahi, F. A. Khan, and A. Samad, "Blood flow and mixing analysis in split-and-recombine micromixer with offset fluid inlets," Am. Soc. Mech. Eng. Fluids Eng. Div. FEDSM, vol. 3, pp. 1-5, 2018, doi: 10.1115/FEDSM201883468.

15. J. Boyd, J. M. Buick, and S. Green, "Analysis of the Casson and Carreau-Yasuda non-Newtonian blood models in steady and oscillatory flows using the lattice Boltzmann method," Phys. Fluids, vol. 19, no. 9, 2007, doi: 10.1063/1.2772250.

16. Dheringe, N., and S. Rahane. "Recent advances in mems sensor technology biomedical mechanical thermo-fluid \& electromagnetic sensors." Int. J. Electron. Commun.Instrum. Eng. Res. Dev 3 (2013): 73-90.

17. F. Abraham, M. Behr, and M. Heinkenschloss, "Shape optimization in steady blood flow: A numerical study of non-Newtonian effects," Comput. Methods Biomech. Biomed. Engin., vol. 8, no. 2, pp. 127-137, 2005, doi: 10.1080/10255840500180799.

18. T. M. Dundi, V. R. K. Raju, and V. P. Chandramohan, "Numerical evaluation of swirl effect on liquid mixing in a passive Tmicromixer," Aust. J. Mech. Eng., vol. 00, no. 00, pp. 1-15, 2019, doi: 10.1080/14484846.2019.1626527. 